\section{Multimodal retinal imaging in a Chinese kindred with familial amyloid polyneuropathy secondary to transthyretin Ile107Met mutation}

${ }^{1}$ Joint Shantou International Eye Center, Shantou University and the Chinese University of Hong Kong, Shantou, China

${ }^{2}$ Department of Ophthalmology and Visual Sciences, the Chinese University of Hong Kong, Hong Kong, China

Correspondence: $\mathrm{H}$ Chen, Joint Shantou International Eye Center, Shantou University \& the Chinese University of Hong Kong, North Dongxia Road, Shantou, Guangdong 515041, PR China. Tel: +86 75488393560 ; Fax: +86 75488393560 . E-mail: drchenhaoyu@ gmail.com

Received: 28 March 2013 Accepted in revised form: 10 December 2013 Published online: 31 January 2014

\begin{abstract}
Objective To investigate the ocular phenotype and gene mutation of a Chinese pedigree with familial amyloid polyneuropathy (FAP) and vitreous amyloidosis.

Methods A Chinese pedigree with familial amyloid polyneuropathy and vitreous amyloidosis was recruited. Combined phacoemulsification, vitrectomy and intraocular lens implantation were performed on the right eye of the index patient. Ophthalmic investigations were performed before and after surgery. The DNA from the pedigree was sequenced for the transthyretin (TTR) gene. Results After vitrectomy, the best-corrected visual acuity of the patient improved from counting finger to 20/20. Red-free confocal ophthalmoscopy demonstrated perifoveal ring and several perivessel white sheaths. Optical coherence tomography (OCT) revealed cotton wool like reflections on the vitreoretinal interface. Electroretinogram and autofluorescence was normal. Amyloid was present in the vitreous specimen. A substitution of $T$ to $G$ at nucleotide 381 in exon 4 of TTR DNA (Ile107Met) was found. This mutation co-segregated with phenotype in the pedigree and was not detected in 200 controls.

Conclusions TTR Ile107Met mutation is associated with vitreous amyloidosis and FAP. OCT and red-free imaging are helpful in identifying amyloid deposits in the retina. Eye (2014) 28, 452-458; doi:10.1038/eye.2014.10; published online 31 January 2014
\end{abstract}

W Lv' ${ }^{1}$, J Chen'1, W Chen ${ }^{1}$, P Hou' ${ }^{1}$, CP Pang ${ }^{1,2}$ and $\mathrm{H} \mathrm{Chen}^{1,2}$
Keywords: vitreous amyloidosis; familial amyloid polyneuropathy; transthyretin; optical coherence tomography

\section{Introduction}

Familial amyloid polyneuropathy (FAP) is a systemic disease transmitted as an autosomal dominant trait, mostly caused by mutation of transthyretin (TTR) gene. It is endemic in Sweden, Japan, and Portugal, but has been sporadically reported worldwide. ${ }^{1}$ The extracellular deposit of amyloid fibrils in sensorimotor peripheral nerves, autonomic nerves, central nervous system, myocardia, kidneys, and vitreous would lead to dysfunction of these tissues, including loss of temperature and pain sensations in lower and upper limbs, autonomic dysfunction, cardiomyopathy, renal dysfunction, gastrointestinal dysfunction, and visual loss. ${ }^{2,3}$ However, not all the organs would be involved in all patients. The spectrum of clinical phenotypes is broad. ${ }^{4}$

In 1978, it was first found that the amyloid fibril protein is related to human prealbumin subunit, which was called TTR. ${ }^{5}$ Val30Met mutation of TTR gene was identified as the cause of FAP. ${ }^{6}$ Since then, more than 100 mutations in TTR gene have been found in FAP. $^{7}$ The prevalence of TTR mutation is different among different populations, which partly explains the ethnic variation in the prevalence of $\mathrm{FAP}^{4}$ 
Vitreous amyloidosis occurs in only $24 \%$ of FAP patients with the TTR Val30Met mutation but in all patients with TTR Tyr114Cys. ${ }^{8}$ Some patients have retinal neovascularization and hemorrhage. ${ }^{9-11}$ Although the vitreous opacity can be removed by vitrectomy, there is recurrence in some cases. ${ }^{12}$ We report the ocular phenotypes using multimodal imaging techniques and genetic analysis in a Chinese kindred with FAP and vitreous amyloidosis.

\section{Materials and methods}

\section{Study subjects}

This study was approved by the Ethics Committee of Joint Shantou International Eye Center and was conducted in accordance with the Declaration of Helsinki. Written consent was obtained from all participants after explaning of the nature of the study. A pedigree (Figure 1c) with FAP involving vitreous was recruited at the Joint Shantou International Eye Center, Shantou, China. Two hundred senile cataract controls without FAP were recruited from surgical inpatients at the hospital. None of the members in the pedigree were included in the controls.

\section{Clinical investigation and management}

A 55-year-old Chinese man presented with a 3-year history of blurred vision with floaters in both eyes. The symptoms were more serious in the right eye. He had developed glove-and-stocking sensation in his hands and feet for 6 years. The best-corrected visual acuity (BCVA) was counting fingers in the right eye and 20/100 in the left eye. Anterior segment was unremarkable except mild cataract in both eyes. Massive dense 'glass-wool' opacity was noted in the vitreous in both eyes (white arrow on slit lamp photo, Figure 2a). Some white fluffy material attached to the posterior capsule was also noted in both eyes (red arrow on slit lamp photo, Figure 2a). No vitreous hemorrhage was found. The fundus could be examined in the right eye, whereas a blurred view was seen in the left eye (fundus photo, Figure 2b). Ultrasound $\mathrm{B}$ scan of the right eye confirmed the presence of vitreous opacity. No other abnormality was detected (Figure 2c). The family history was positive for FAP in four family members (Figure 1c). The patient's elder sister had received vitrectomy for vitreous opacities 3 years ago. She was unable to walk, did not gain weight, and had alternating diarrhea and constipation for 2 years.
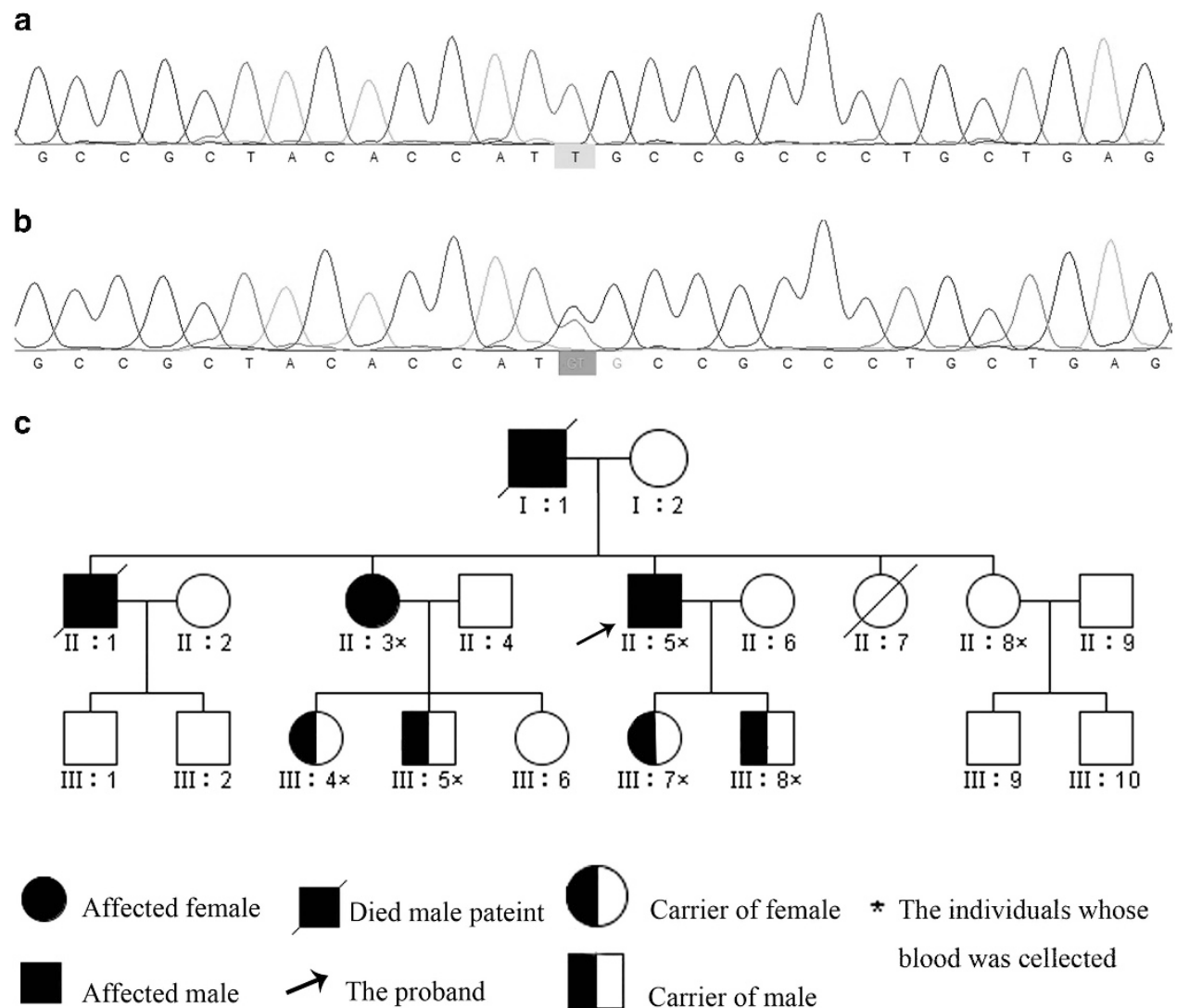

Figure 1 Mutation analysis of a Chinese family with familial amyloid polyneuropathy. (a) Normal sequence of TTR gene. (b) The base $\mathrm{T}$ is substituted by G at the position 381 in TTR cDNA (red square). (c) Co-segregation of the mutation in the pedigree. A full color version of this figure is available at the Eye journal online. 

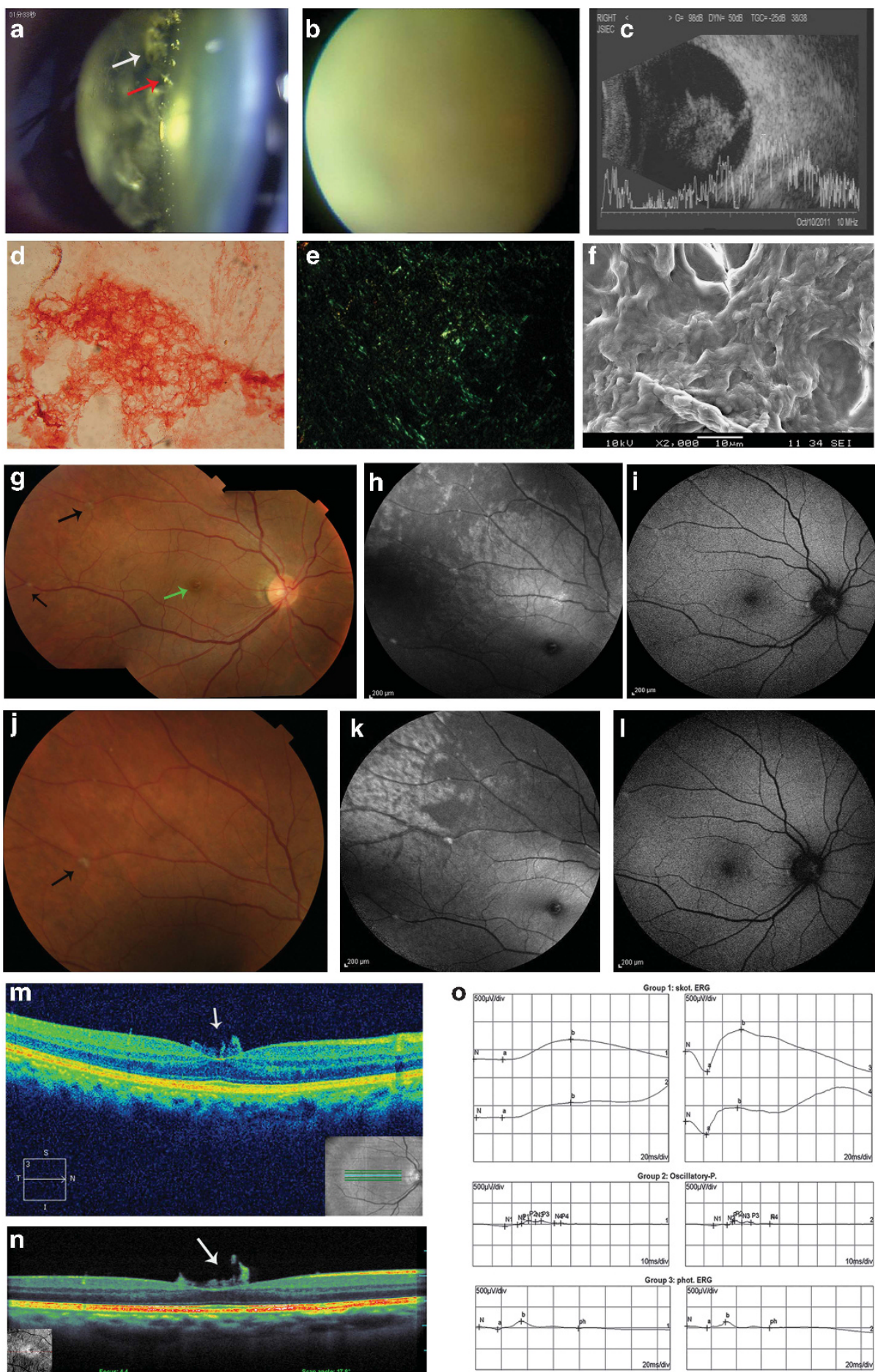

Figure 2 Clinical and pathological imaging of the proband's right eye. (a-c) Preoperative images. (a) Glass-wool like material in the vitreous (white arrow), and some punctuate white dots attached to the posterior capsule of the lens (red arrow). (b) The fundus is not visible. (c) Ultrasound examination showed low-level echo in the vitreous. (d-f) Pathological results. (d) The vitreous was positively stained with Congo-red. (e) The amyloid material showed the color of apple green under polarized light. (f) Amyloid fibrils under electron microscope. ( $g, h, i, m$, and o) Images at 1 week after operation. (j, k, l, and n) Images at 1 year after operation. (g and j) Color fundus photography showed perifoveal (green arrow) and perivessel amyloid deposit (black arrow). (i and l) Autofluorescence was normal. (h) Red-free confocal imaging demonstrated hyper-reflection at perifoveal perivessel deposit and at temporal retina 1 month after operation. (k) One year after operation, red-free confocal imaging showed enlargement of the area of high reflection. (m and $\mathrm{n}$ ) Optical coherence tomography showed high reflective deposit at the vitreofoveal interface. (o) ERG was normal in both eyes 1 week after operation. 
Furthermore, the patient's father and elder brother had similar gastrointestinal symptoms in addition to muscular dystrophy and had died within 10 years after becoming totally blind. The patient's younger sister, 49 years old at the time of this study, remained healthy. The characteristics of the family members are listed in Table 1.

The patient received combined phacoemulsification, vitrectomy, and intraocular lens implantation on his right eye. The vitreous sample collected during vitrectomy was examined with Congo red staining and processed for electron microscopy. Fundus photography (TRC-50DX, Topcon, Tokyo, Japan), spectral domain optical coherence tomography (OCT, HD 5 Line Raster mode in Cirrus HDOCT, Zeiss Meditec, Oberkochen, Germany and Line Scanning mode in Optos OCT/SLO, Optos, Dunfermline, UK), autofluorescence and red-free imaging with laser scanning confocal ophthalmoscope (HRA2, Heidelberg Engineering, Heidelberg, Germany), and electroretinogram (ERG, RETIport32, Roland Instruments, Wiesbaden, Germany) were performed after operation.

\section{Mutation screening}

Peripheral blood was collected from seven members of the family (II:3, II:5, II:8, III:4, III:5, III:7, III:8) and all senile cataract controls. Genomic DNA was extracted using the QIAmp Blood kit (Qiagen, Hilden, Germany). Polymerase chain reaction (PCR) amplication was performed using the GeneAmp PCR System 9700 (ABI, Foster City, CA, USA) following cycling conditions: 1 cycle of $95^{\circ} \mathrm{C}, 5 \mathrm{~min} ; 25$ cycles of $95^{\circ} \mathrm{C}$ for $30 \mathrm{~s}, 57^{\circ} \mathrm{C}$ for $30 \mathrm{~s}$, and $72{ }^{\circ} \mathrm{C}$ for $30 \mathrm{~s}, 72{ }^{\circ} \mathrm{C}$ for $10 \mathrm{~min}$. The reaction

Table 1 Demographic details of the subjects

\begin{tabular}{lclcc}
\hline ID & Age (year) & Gender & BCVA od & BCVA os \\
\hline II: 3 & 64 & Female & Counting figure & Counting figure \\
II: 5 & 55 & Male & Counting figure & $20 / 100$ \\
II: 8 & 49 & Female & $20 / 25$ & $20 / 25$ \\
III: 3 & 40 & Female & $20 / 20$ & $20 / 20$ \\
III: 4 & 30 & Male & $20 / 20$ & $20 / 20$ \\
III: 6 & 28 & Female & $20 / 20$ & $20 / 20$ \\
III: 7 & 26 & Male & $20 / 20$ & $20 / 20$ \\
\hline
\end{tabular}

Abreviation: BCVA, best-corrected visual acuity. mixture was $22.15 \mu$ l deionized water, $3 \mu \mathrm{l} 10 \times$ buffer, $0.6 \mu \mathrm{l} 2.5 \mu \mathrm{mol} / \mu \mathrm{l} \mathrm{dNTP,} 0.6 \mu \mathrm{l} 10 \mu \mathrm{mol} / \mu \mathrm{l}$ of each forward/reverse primer, $0.75 \mu \mathrm{l}$ Taq polymerase, $40 \mathrm{ng}$ DNA template in a final volume of $30 \mu \mathrm{l}$. The primers for PCR were listed in Table 2. All four TTR exons were directly sequenced in both forward and reverse by 3130XL genetic analyzer (ABI). Data were collected and analyzed using novoSNP sequencing analysis software (VIB, Gent, Belgium). Mutation naming followed the nomenclature recommended by the Human Genomic Variation Society (HGVS).

\section{Results}

\section{Clinical investigation and management}

The BCVA of the right eye of the index cases was 20/20 at 1 week and 20/25 at a 1-year follow-up. The vitreous specimen was positive for atypical protein or amyloids by Congo red staining (Figure $2 \mathrm{~d}$ ) and had an apple green birefringence under the polarization microscope (Figure 2e). Electron microscopy revealed amyloid fibrils in the vitreous (Figure 2f). Under slit lamp biomicroscopy and fundus photography, a perifoval ring and white deposits on some major vessels were found 1 week after operation (Figure 2g). The lesions remained unchanged 1 year after operation (Figure 2f). Autofluorescence was unremarkable 1 week and 1 year after operation, respectively (Figures $2 \mathrm{i}$ and 1 ). On confocal red-free imaging, besides the more striking perifoval ring and perivascular deposit, a high reflection signal was noted on the temporal retina (Figure 2h). At 1 year after operation, the area of high reflection expanded (Figure 2k). OCT showed that the perifoveal ring was the high reflective deposit at vitreoretinal interface of the macula (Figures $2 \mathrm{~m}$ and $\mathrm{n}$ ). The ERG was normal in both eyes (Figure 2o).

\section{Mutation analysis}

Gene sequencing analysis detected a heterozygous single-nucleotide change, (c.381T $>\mathrm{G})$ in exon 4 of the TTR gene in the proband (II:5) (Figure 1b). This substitution resulted in change of the encoded aminoacid residue from isoleucine (Ile) to methionine (Met) at

Table 2 The sequences of primers for direct sequencing of TTR gene

\begin{tabular}{lll}
\hline Exon & Forward $\left(5^{\prime} \rightarrow 3^{\prime}\right)$ & Reverse $\left(5^{\prime} \rightarrow 3^{\prime}\right)$ \\
\hline 1 & AATGTTCCGATGCTCTAATCTAATCTAATCTC & GACTCACTTCTACTTCATTTAGCG \\
2 & TGTGTAATTCTTGTTTCGCTCC & CTTCAGTGGGCATACTTGAC \\
3 & CTACTTCTGACTTAGTTGAGG & CCTCGAAGGTCTGTAGACTC \\
4 & CTCTGGTGGAAATGGATCTG & GCTATTTGCTGAAGGTTA \\
\hline
\end{tabular}


codon 107. The mutation was also found in the proband's offsprings (III:7, III:8), elderly sister (II:3), and the elderly sister's offsprings (III:4, III:5). The mutation was absent in the proband's younger sister (II:8) who was 49 years old and was healthy. All the subjects of the third generation carrying the mutation (III:4, III:5, III:7, III:8) remained asymptomatic. They were younger than 40 years. The mutation was not found in any of the 200 senile cataract controls. No other mutation was detected at TTR exon in the affected family members.

\section{Discussion}

In this study, we reported a Chinese family with FAP involving vitreous caused by a TTR gene mutation, c.381T $>$ G, Ile107Met. We also demonstrated that amyloid deposits could be seen on fundus photography, confocal red-free imaging, and spectral domain OCT. Confocal red-free imaging showed the deposit on the vitreous-retinal interface and revealed intraretinal reflective lesions.

The TTR gene is located at $18 \mathrm{q} 11.2-12$, is small $(7 \mathrm{~kb})$, and contains four exons. It encodes a 127-residue polypeptide chain. At present, at least 113 mutations in the TTR gene have been identified associated with amyloidosis. ${ }^{2}$ Among them, Val30Met is the most common mutation. It is almost the only variant detected in FAP patients in Portugal, Brazil, and Sweden. By contrast, many different TTR variants are reported in other ethnicities, such as Japan and France. ${ }^{2}$ In Chinese, several variations have been reported, some of them not related to vitreous amyloidosis, such as Val30Met, ${ }^{13}$ Val30Ala, ${ }^{14}$ Val32Ala, ${ }^{15}$ Phe33Val, ${ }^{13}$ Leu55Pro, ${ }^{16}$ Thr59Lys, ${ }^{17}$ Arg104His, ${ }^{17}$ Gly67Glu, ${ }^{18}$ Ala97Ser, ${ }^{19,20}$ Tyr114Cys. ${ }^{21}$ Some were associated with vitreous involvement, such as Lys35Thr, ${ }^{22}$ Leu55Arg, ${ }^{22}$ Arg54Gly, ${ }^{23}$ Gly83Arg, ${ }^{24}$ and Gly103Arg. ${ }^{25}$ In our study, the Ile107Met mutation was identified in two patients in a family. It was absent in a middle-aged healthy sister of the index case as well as the control subjects, suggesting that it is the cause of FAP and vitreous amyloidosis. The Ile107Met mutation has been reported in Caucasians in two conference papers. ${ }^{26,27}$ The observation of TTR Ile107Met mutation in our Chinese pedigree underscores the homogeneity of this mutation as a cause of FAP involving vitreous across ethnic divisions. The phenotype in our case is similar to the previously reported cases, including the presence of vitreous opacities as well as polyneuropathy. However, there was no detail on ocular phenotype reported in the previous papers. $^{26,27}$

Slit lamp biomicroscopy and fundus photography showed a perifoval ring and white deposits on major vessels in our case (Figure $2 \mathrm{~g}$ ). The perifoval ring and perivascular deposit were more striking on confocal redfree imaging. Besides, a highly reflective signal was noted on the temporal retina (Figure $2 \mathrm{~h}$ ). These lesions may reperesent intraretinal deposits of amyloid fibers. The amyloid fibers are organized in a parallel pattern, ${ }^{28}$ similar to the retinal nerve fiber layer, which is hyperreflective on red-free imaging of laser scanning confocal ophthalmoscope. On spectral domain OCT, the perifoval ring was seen as a highly reflective deposit on the vitreoretinal interface at the macula. The red-free imaging in confocal ophthalmoscope and OCT can be used for early detection of amyloidosis as well as to monitor the progress of the disease.

It was previously reported that the needle-shaped deposits perpendicular to the retinal surface are oriented towards the vitreous on the OCT. ${ }^{11}$ These deposits were extensively found on the macula in the reported literature; ${ }^{11}$ however, they were limited to the perifoval region in our case. This observation may be attributed to the fact that most of these deposits were removed during vitrectomy. Other findings such as retinal hemorrhages that have been reported previously ${ }^{9-11}$ were absent in our case. These deviations suggested clinical heterogeneity of vitreous amyloidosis.

It was reported that $24 \%$ of the vitreous opacities recurred after vitrectomy. ${ }^{29}$ Extensive vitrectomy can prevent the recurrence of vitreous amyloidosis. ${ }^{12}$ In our case, the lesions remain unchanged on OCT at 1 year postoperatively. However, we observed an area of hyperreflective lesion on red-free confocal ophthalmoscope. It is possible that a thorough vitrectomy prevented the accumulation of amyloid fibers in the vitreous, but the amyloid fiber may still deposit intraretinally. Longer follow-up duration on a large number of patients is needed to verify this hypothesis.

The ERG result of our patients was normal. The TTR is an indirect carrier of retinol through binding to the retinol-binding protein. ${ }^{30}$ However, good visual acuity of patients with TTR-related vitreous amyloidosis had been previously reported. ${ }^{8,12}$ The visual acuity of the index case in our study recovered after the surgery. Further, the TTR knockout mice did not show any abnormalities ${ }^{31}$ in ocular structure or ERG compared with wild-type mice. ${ }^{32}$ These evidences suggest that loss of TTR function does not affect the physiological role of retinol in vision.

RPE is the major source of TTR in the retina. ${ }^{33}$ Panretinal laser photocoagulation prevents the amyloid deposits in the vitreous and on the retinal surface, through damaging the retinal pigment epithelium. ${ }^{34}$ However, the autofluorescence in our case was normal, suggesting that the TTR mutation did not cause the accumulation of lipofuscin in the RPE.

It would have been interesting to compare the retinal thickness during the follow-up. However, we recognized 
that the two OCT images were obtained with different instruments. The OCT immediately after surgery was taken with Zeiss Cirrus OCT, while the OCT at 1-year post vitrectomy was taken with an Optos OCT/SLO system. Different algorithm of image processing and analysis was used in different instruments. Therefore, we could not compare the curvature and thickness of retinal layers of images from different instruments.

In conclusion, we reported a Chinese pedigree of familial amyloid polyneuropathy resulting from TTR Ile107Met mutation. Confocal red-free imaging and OCT demonstrated deposits of amyloid fibers in the retina. Autofluorescence and ERG was normal, suggesting that the mutation did not affect the visual function of the retina.

\section{Summary}

\section{What was known before}

- Familial amyloid polyneuropathy (FAP) is an autosomal dominant disease caused by mutation of transthyretin (TTR) gene. Up to now, more than 100 mutations in TTR gene have been found in FAP. Clinical heterogeneity was also found. Even in patients with vitreous amyloidosis, the spectrum of clinical phenotypes was broad.

\section{What this study adds}

- Our study found that TTR Ile107Met mutation is associated with vitreous amyloidosis and FAP in a Chinese pedigree. Optical coherence tomography and red-free confocal ophthalmoscopy can demonstrate deposits in the retina and help monitoring progress of disease. Autofluorescence and electroretinogram were normal.

\section{Conflict of interest}

The authors declare no conflict of interest.

\section{Acknowledgements}

This study was supported by the National Nature Science Foundation of China (30901646 and 81170853), Guangdong Science and Technology Project (2011B031300013), Guangdong Medical Research Foundation (B2010230).

\section{References}

1 Ando Y, Nakamura M, Araki S. Transthyretin-related familial amyloidotic polyneuropathy. Arch Neurol 2005; 62(7): 1057-1062.

2 Plante-Bordeneuve V, Said G. Familial amyloid polyneuropathy. Lancet Neurol 2011; 10(12): 1086-1097.

3 Sandgren O. Ocular amyloidosis, with special reference to the hereditary forms with vitreous involvement. Surv Ophthalmol 1995; 40(3): 173-196.
4 Ikeda S, Nakazato M, Ando Y, Sobue G. Familial transthyretin-type amyloid polyneuropathy in Japan: clinical and genetic heterogeneity. Neurology 2002; 58(7): 1001-1007.

5 Costa PP, Figueira AS, Bravo FR. Amyloid fibril protein related to prealbumin in familial amyloidotic polyneuropathy. Proc Natl Acad Sci USA 1978; 75(9): 4499-4503.

6 Saraiva MJ, Birken S, Costa PP, Goodman DS. Family studies of the genetic abnormality in transthyretin (prealbumin) in Portuguese patients with familial amyloidotic polyneuropathy. Ann N Y Acad Sci 1984; 435: 86-100.

7 Benson MD, Kincaid JC. The molecular biology and clinical features of amyloid neuropathy. Muscle Nerve 2007; 36(4): 411-423.

8 Koga T, Ando E, Hirata A, Fukushima M, Kimura A, Ando $Y$ et al. Vitreous opacities and outcome of vitreous surgery in patients with familial amyloidotic polyneuropathy. Am J Ophthalmol 2003; 135(2): 188-193.

9 Savage DJ, Mango CA, Streeten BW. Amyloidosis of the vitreous. Fluorescein angiographic findings and association with neovascularization. Arch Ophthalmol 1982; 100(11): 1776-1779.

10 Dunlop AA, Graham SL. Familial amyloidotic polyneuropathy presenting with rubeotic glaucoma. Clin Experiment Ophthalmol 2002; 30(4): 300-302.

11 Hattori T, Shimada H, Yuzawa M, Kinukawa N, Fukuda T, Yasuda N. Needle-shaped deposits on retinal surface in a case of ocular amyloidosis. Eur J Ophthalmol 2008; 18(3): 473-475.

12 Beirao NM, Matos E, Beirao I, Costa PP, Torres P. Recurrence of vitreous amyloidosis and need of surgical reintervention in Portuguese patients with familial amyloidosis ATTR V30M. Retina 2011; 31(7): 1373-1377.

$13 \mathrm{Li} \mathrm{YF,} \mathrm{Ng} \mathrm{H}$, Sun IU, Leong W. Clinical and genetic analysis of three families with familiar amyloid polyneuropathy. Chin Med Sci J 2008; 23(4): 230-233.

14 Mak CM, Lam CW, Fan ST, Liu CL, Tam SC. Genetics of familial amyloidotic polyneuropathy in a Hong Kong Chinese kindred. Acta Neurol Scand 2003; 107(6): 419-422.

15 Pica EC, Pramono ZA, Verma KK, San LP, Chee YW. A novel transthyretin mutation V32A in a Chinese man with late-onset amyloid polyneuropathy. Muscle Nerve 2005; 32(2): 223-225.

16 Chou CT, Lee CC, Chang DM, Buxbaum JN, Jacobson DR. Familial amyloidosis in one Chinese family: clinical, immunological, and molecular genetic analysis. J Intern Med 1997; 241(4): 327-331.

17 Lim A, Prokaeva T, Connor LH, Falk RH, Skinner M, Costello CE. Identification of a novel transthyretin Thr59Lys/Arg104His. A case of compound heterozygosity in a Chinese patient diagnosed with familial transthyretin amyloidosis. Amyloid 2002; 9(2): 134-140.

18 Mak CM, Kwong YL, Lam CW, Chan SC, Lo CM, Fan ST et al. Identification of a novel TTR Gly67Glu mutant and the first case series of familial transthyretin amyloidosis in Hong Kong Chinese. Amyloid 2007; 14(4): 293-297.

19 Lachmann HJ, Booth DR, Bybee A, Hawkins PN. Transthyretin Ala97Ser is associated with familial amyloidotic polyneuropathy in a Chinese-Taiwanese family. Hum Mutat 2000; 16(2): 180.

20 Liu YT, Lee YC, Yang CC, Chen ML, Lin KP. Transthyretin Ala97Ser in Chinese-Taiwanese patients with familial 
amyloid polyneuropathy: genetic studies and phenotype expression. J Neurol Sci 2008; 267(1-2): 91-99.

21 Zhang Y, Deng YL, Ma JF, Zheng L, Hong Z, Wang ZQ et al. Transthyretin-related hereditary amyloidosis in a Chinese family with TTR Y114C mutation. Neurodegener Dis 2011; 8(4): 187-193.

22 Long D, Zeng J, Wu LQ, Tang LS, Wang HL, Wang H. Vitreous amyloidosis in two large mainland Chinese kindreds resulting from transthyretin variant Lys35Thr and Leu55Arg. Ophthalmic Genet 2011; 33(1): 28-33.

23 Shi YN, Li J, Hu J, Sun LJ, Li HJ, Shi R et al. A new Arg54Gly transthyretin gene mutation associated with vitreous amyloidosis in Chinese. Yan Ke Xue Bao 2011; 26(4): 230-238.

24 Chen L, Lu L, Zhang P, Li Y, Lin J. Transthyretin Arg-83 mutation in vitreous amyloidosis. Yan Ke Xue Bao 2008; 24(1): 65-67.

25 Xie Y, Zhao Y, Zhou JJ, Wang X. Identification of a TTR gene mutation in a family with hereditary vitreous amyloidosis. Zhonghua Yi Xue Yi Chuan Xue Za Zhi 2012; 29(1): 13-15.

26 Altland K. Common molecular characteristics of amyloidogenic TTR mutations. The 4th International Symposium on FAP and Other TTR Related Disorders Umeå, Sweden, 1999.

27 Spencer B, Connors LH, Prokaeva T, Soohoo P, Hara CO. M S. recent novel and rare mutations in a clinic population of patients with amyloidosis. In: Skinner M, Berk JL,
Connors LH, DC S (eds). 11th International Symposium on Amyloidosis. Taylor \& Francis, 2008, pp 199-201.

28 Sunde M, Blake C. The structure of amyloid fibrils by electron microscopy and X-ray diffraction. Adv Protein Chem 1997; 50: 123-159.

29 Doft BH, Machemer R, Skinner M, Buettner H, Clarkson J, Crock J et al. Pars plana vitrectomy for vitreous amyloidosis. Ophthalmology 1987; 94(6): 607-611.

30 Raz A, Goodman DS. The interaction of thyroxine with human plasma prealbumin and with the prealbuminretinol-binding protein complex. J Biol Chem 1969; 244(12): 3230-3237.

31 Wei S, Episkopou V, Piantedosi R, Maeda S, Shimada K, Gottesman ME et al. Studies on the metabolism of retinol and retinol-binding protein in transthyretin-deficient mice produced by homologous recombination. J Biol Chem 1995; 270(2): 866-870.

32 Bui BV, Armitage JA, Fletcher EL, Richardson SJ, Schreiber G, Vingrys AJ. Retinal anatomy and function of the transthyretin null mouse. Exp Eye Res 2001; 73(5): 651-659.

33 Dwork AJ, Cavallaro T, Martone RL, Goodman DS, Schon EA, Herbert J. Distribution of transthyretin in the rat eye. Invest Ophthalmol Vis Sci 1990; 31(3): 489-496.

34 Kawaji T, Ando Y, Hara R, Tanihara H. Novel therapy for transthyretin-related ocular amyloidosis: a pilot study of retinal laser photocoagulation. Ophthalmology 2010; 117(3): 552-555. 